\title{
Potential use of glutathione as a treatment for Parkinson's disease
}

\author{
HAI-LI WANG ${ }^{1 *}$, JUN ZHANG $^{1 *}$, YU-PING LI $^{2}$, LUN DONG $^{2}$ and YING-ZHU CHEN ${ }^{3}$ \\ ${ }^{1}$ Department of Clinical Medicine, Dalian Medical University, Dalian, Liaoning 116000; Departments of \\ ${ }^{2}$ Neurosurgery and ${ }^{3}$ Neurology, Clinical Medical College of Yangzhou University, Yangzhou, Jiangsu 225001, P.R. China
}

Received April 14, 2020; Accepted October 16, 2020

DOI: $10.3892 /$ etm.2020.9557

\begin{abstract}
The aim of the present study was to assess the efficacy and safety of glutathione (GSH) for the treatment of Parkinson's disease (PD). The PubMed, Cochrane Library, OvidSP, Web of Science, China Science and Technology Journal Database, Chinese National Knowledge Infrastructure and China Wanfang Standards Database databases were systematically searched from the inception dates to October 1st, 2019, using the key words'glutathione' or 'GSH' and 'Parkinson' or 'Parkinson's disease' or 'PD'. The quality of the included articles was assessed using the bias risk assessment tool of the Cochrane systematic evaluator manual (version 5.1.0). Pooled analysis of the relevant data was performed using RevMan 5.3 software and subgroup analysis was performed to determine the impact of the dosage (300 vs. $600 \mathrm{mg}$ ) on the outcome measures. A total of seven randomized controlled trials involving 450 participants were included in the meta-analysis. The results of the present study indicated a statistically significant difference between the GSH and control groups, in terms of the Unified Parkinson's Disease Rating Scale (UPDRS) III [standard mean difference (SMD), -0.48; 95\% CI, -(0.88-0.08); $\mathrm{P}=0.02]$ and GSH peroxidase (SMD, 1.88; 95\% CI, 0.52-3.24; $\mathrm{P}=0.007)$. However, the differences in the UPDRS I (SMD, -0.04; 95\% CI, -0.25-0.16; $\mathrm{P}=0.70$ ) and UPDRS II (SMD, 0.03; 95\% CI, -0.17-0.24; $\mathrm{P}=0.77$ ) score and in side effects were not statistically significant between the groups. Subgroup analyses revealed that the dosage (300 vs. $600 \mathrm{mg}$ ) was an influencing factor for UPDRS III. The present study demonstrated that GSH may mildly improve motor scores in PD, but not at the expense of increased adverse events.
\end{abstract}

Correspondence to: Professor Ying-Zhu Chen, Department of Neurology, Clinical Medical College of Yangzhou University, 98 Nantong West Road, Yangzhou, Jiangsu 225001, P.R. China E-mail: yzchendr@163.com

*Contributed equally

Key words: glutathione, meta-analysis, randomized controlled trial, Parkinson's disease

\section{Introduction}

Parkinson's disease (PD) is a progressive neurodegenerative disease associated with aging, which is characterized by the selective loss of nigrostriatal dopaminergic neurons (1-3). PD is the second most common neurodegenerative disease in the world with a prevalence that is estimated to reach between 8.7 and 9.3 million by 2030 (4). To date, the pathophysiology of PD remains to be fully elucidated, though studies indicate that oxidative stress may be one of the mechanisms contributing to $\mathrm{PD}$ (5). There is currently no cure for PD; thus, further research into the development of novel treatment strategies is critical (6). Increasing evidence has demonstrated that oxidative stress has an important role in the events contributing to the degeneration of dopaminergic neurons (7), and that redox reactions are a possible source of oxidative stress in nigral dopaminergic neurons (8). Glutathione (GSH) is a ubiquitous thiol tripeptide that protects against oxidative stress-induced damage by neutralizing reactive oxygen species (5). GSH deficiency has been identified as an early event in the progression of PD (9). Therefore, supplementing GSH may effectively improve the symptoms of PD. In recent years, a number of clinical trials have sought to investigate the effects of GSH treatment for PD (10-12). Regrettably, the sample size of these studies was small and the clinical evidence is insufficient (10-12). To the best of our knowledge, no previous meta-analyses have assessed the efficacy and safety of GSH in patients with PD. Hence, in the present study, a meta-analysis was performed with the aim of providing medical evidence-based support for GSH treatment in these patients.

\section{Materials and methods}

Search strategy. To identify eligible studies, a primary search was conducted using electronic databases (PubMed, Cochrane Library, OvidSP, Web of Science, China Science and Technology Journal Database, Chinese National Knowledge Infrastructure and China Wanfang Standards Database) from the inception dates to October 1st, 2019, using the keywords 'glutathione' or 'GSH' and 'Parkinson' or 'Parkinson's disease' or 'PD'. Specific retrieval strategies were adjusted according to different databases. The procedure was concluded by: i) The perusal of the reference sections of all relevant studies; ii) a manual search for GSH in key journals and abstracts from the major annual meetings in the field of PD; and iii) contact with experts to request unpublished data. The primary search 
Table I. Characteristics of the included trials and participants.

\begin{tabular}{|c|c|c|c|c|c|c|c|}
\hline First author (year) & Design & Follow-up & $\begin{array}{l}\text { Age (years) } \\
\text { GSH/Control }\end{array}$ & $\begin{array}{c}\text { Participants } \\
\text { (males/females) } \\
\text { GSH/Control }\end{array}$ & $\begin{array}{c}\text { Intervention } \\
\text { Route, dose, frequency }\end{array}$ & Outcomes & (Refs.) \\
\hline Hauser (2009) & $\mathrm{RCT}$ & $4 w$ & $\begin{array}{l}62.6+7.9 / \\
65.9+12.6\end{array}$ & $(5 / 5) /(6 / 4)$ & $\begin{array}{l}\text { Intravenous push, } \\
1400 \mathrm{mg}, \mathrm{Qd}\end{array}$ & $\begin{array}{l}\mathrm{A} ; \mathrm{B} ; \\
\mathrm{C} ; \mathrm{E}\end{array}$ & (10) \\
\hline Mischley (2017) & $\mathrm{RCT}$ & $3 \mathrm{~m}$ & $\begin{array}{c}60.9+11 / \\
60.9+11\end{array}$ & $11 ; 14 / 14$ & $\begin{array}{l}\text { Intranasal administration, } \\
300 \mathrm{mg} \text { or } 600 \mathrm{mg}, \mathrm{Qd}\end{array}$ & $\mathrm{A} ; \mathrm{B} ; \mathrm{C}$ & (11) \\
\hline Mischley (2015) & $\mathrm{RCT}$ & $3 \mathrm{~m}$ & - & $10 ; 10 / 10$ & $\begin{array}{l}\text { Intranasal administration, } \\
300 \mathrm{mg} \text { or } 600 \mathrm{mg} \text {, Qd }\end{array}$ & $\begin{array}{l}\mathrm{A} ; \mathrm{B} ; \\
\mathrm{C} ; \mathrm{E}\end{array}$ & (12) \\
\hline Bao (2018) & $\mathrm{RCT}$ & $4 \mathrm{~m}$ & $\begin{array}{l}64.6+8.2 / \\
65.1+9.6\end{array}$ & $(56 / 44) /(55 / 45)$ & $\begin{array}{l}\text { Intravenous drip, } 600 \mathrm{mg} \text {, } \\
\text { Bid }\end{array}$ & $\begin{array}{l}\mathrm{A} ; \mathrm{B} ; \\
\mathrm{C} ; \mathrm{D}\end{array}$ & (14) \\
\hline Bao (2003) & $\mathrm{RCT}$ & $6 w$ & $\begin{array}{l}61.41+9.68 / \\
58.87+7.94\end{array}$ & $(14 / 16) /(16 / 14)$ & $\begin{array}{l}\text { Intravenous drip, } 600 \mathrm{mg} \text {, } \\
\text { Bid }\end{array}$ & $\mathrm{D}$ & (15) \\
\hline $\mathrm{Hu}$ (2019) & $\mathrm{RCT}$ & $21 \mathrm{~d}$ & $\begin{array}{c}66.8+6.9 / \\
70.7+7\end{array}$ & $(17 / 15) /(18 / 13)$ & $\begin{array}{l}\text { Intravenous drip, } \\
1200-1400 \mathrm{mg} \text {, Qd }\end{array}$ & $\begin{array}{l}\mathrm{A} ; \mathrm{B} ; \\
\mathrm{C} ; \mathrm{D} ; \mathrm{E}\end{array}$ & (16) \\
\hline Zhang (2005) & $\mathrm{RCT}$ & $4 \mathrm{~m}$ & $\begin{array}{c}56+4.5 / \\
57+4.9\end{array}$ & $(12 / 7) /(11 / 8)$ & $\begin{array}{l}\text { Intravenous drip, } \\
600 \mathrm{mg} \text {, Bid }\end{array}$ & $\mathrm{D}$ & (17) \\
\hline
\end{tabular}

GSH, reduced glutathione; RCT, randomized controlled trial; Bid, bis in die; Qd, quaque die; w, weeks; m, months; d, days; UPDRS, Unified Parkinson's Disease Rating Scale; A, UPDRS I; B, UPDRS II; C, UPDRS III; D, glutathione peroxidase; E, adverse events; m, months; w, weeks.

was completed by independent investigators (HLW and JZ) and any discrepancies were resolved by consultation with an investigator (YZC) not involved in the initial procedure.

Inclusion criteria. The inclusion criteria for the present study were as follows: i) Participants were clinically diagnosed with PD; ii) GSH was administered as an intervention treatment; iii) patients treated with GSH were directly compared with a non-GSH or placebo group; iv) outcomes were determined using the Unified Parkinson's Disease Rating Scale (UPDRS) and/or GSH peroxidase (GSH-Px) and/or related adverse events (AEs); and v) the study was a published randomized controlled trial (RCT).

Exclusion criteria. Articles fulfilling the following criteria were excluded from the present study: i) Randomized trials without a placebo or control group; ii) studies lacking original data; and iii) abstracts, conference papers, letters or comments.

Quality assessment. The risk of bias in the included studies was assessed by two independent reviewers (WHL and JZ) using the Cochrane Handbook for Systematic Reviews of Interventions (13). Bias was evaluated in the following seven domains: i) Random sequence generation; ii) allocation concealment; iii) blinding of participants and personnel; iv) blinding of outcome assessment; v) incomplete outcome data; vi) selective outcome reporting; and vii) other bias, of which random sequence generation, blinding of participants and personnel, and blinding of outcomes assessment were of most interest. Any disagreements were resolved by discussion among all of the reviewers. The risk of bias in each domain was rated as low, unclear or high, according to methods used to ensure the minimization of each form of bias. Using the following methods, individual studies were categorized as having low, high or unclear risk of bias: i) Low risk of bias (plausible bias unlikely to markedly alter the findings) if the risk of bias was low in all domains; ii) unclear risk of bias (plausible bias that raises certain doubt about the results) if the risk of bias was unclear in one or more domains; or iii) high risk of bias (plausible bias that seriously weakens confidence in the results) if a high risk of bias was present in one or more domains. Any disagreements were resolved through a discussion within the entire review team.

Data extraction. Data were extracted by two independent reviewers (WHL and YPL) using a predefined data extraction method. Disagreements were resolved by discussion or consensus with a third independent author (CYZ). The extracted data included the first author, study characteristics (i.e. year, duration and design), participant characteristics (i.e. age, sample size and systemic therapy) and outcomes (UPDRS/GSH-Px/related AEs). For studies with insufficient information, the reviewers contacted the corresponding authors where possible to acquire the data.

Statistical analysis. When conditions permitted, the study was divided into three arms based on the administered dose of GSH used to obtain the two-arm data $(300 \mathrm{mg} / \mathrm{d}$ groups vs. control groups, and $600 \mathrm{mg} / \mathrm{d}$ groups vs. control groups). Dichotomous data were analyzed using the risk ratios (RRs) with $95 \%$ confidence intervals (CIs). When the result unit, measurement method or measurement time was inconsistent, continuous outcome measurements were analyzed using standard mean differences (SMDs) with 95\% CIs; 95\% CIs 


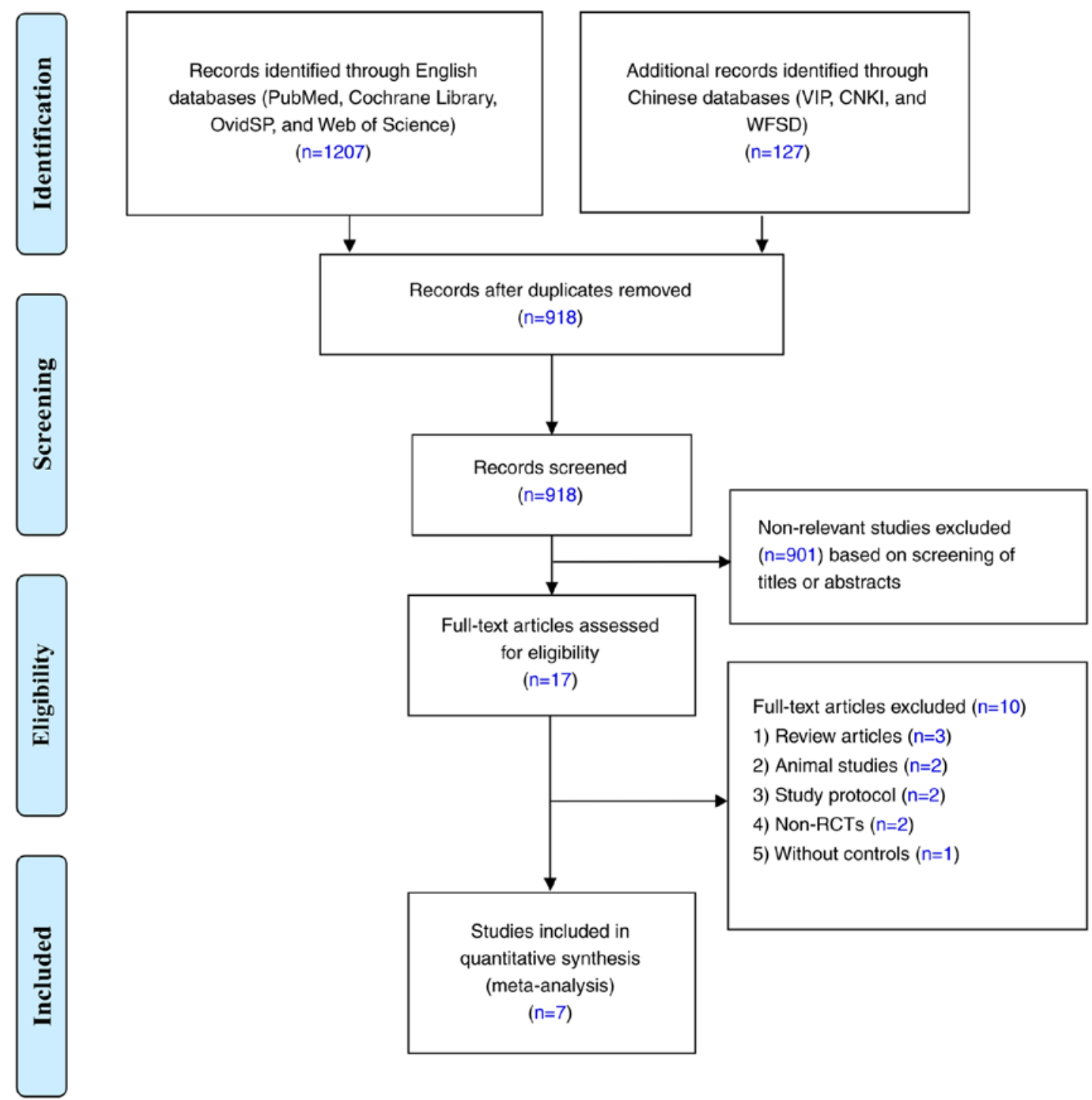

Figure 1. Literature search and screening process. RCT, randomized controlled trial; VIP, China Science and Technology Journal Database; CNKI, Chinese National Knowledge Infrastructure; WFSD, China Wanfang Standards Database.

were calculated using the inverse variance (IV) statistical method. I-square $\left(\mathrm{I}^{2}\right)$ statistics and the $\mathrm{Q}$ test were performed to assess the impact of study heterogeneity on the results of the meta-analysis. According to the Cochrane review guidelines (13), if severe heterogeneity was present at $\mathrm{P}<0.1$ or $\mathrm{I}^{2}>50 \%$, the random-effects model was chosen; otherwise, the fixed-effects model was used. Subgroup analyses were performed according to GSH dose.

\section{Results}

Search results. According to the aforementioned retrieval strategy, a total of 1,334 related articles were initially retrieved and 918 studies were retained after 416 papers with duplicate data were excluded. Of the identified articles, 901 that did not meet the inclusion criteria were excluded after reading the title and abstract. Of the remaining 17 studies (which were evaluated for applicability by reading the full text), a further 10 were omitted per the exclusion criteria, leaving a total of 7 included studies (10-12,14-17). A flow diagram of the screening process is depicted in Fig. 1. The age distribution of the patients within these studies was 43-84 years and the included studies were published between 2003 and 2019. The studies primarily reported on the outcomes of UPDRS, GSH-Px and related AEs. The specific basic characteristics of the included studies are listed in Table I.

Quality assessment. The quality of the included RCTs was assessed according to the Cochrane Handbook (Figs. 2 and 3) (13). In the category random sequence generation, the seven studies had a low risk. There were two articles with sufficient allocation concealment, while the allocation and concealment schemes of the other five articles were not clear. Furthermore, performance bias of three studies were low-risk and four articles were unclear. There were two papers with low detection bias, while another five articles were rated as unclear with regard to this bias. In terms of incomplete data, seven articles were all rated as having low risk, and the risk of selective reporting was low in five articles and was high in two 


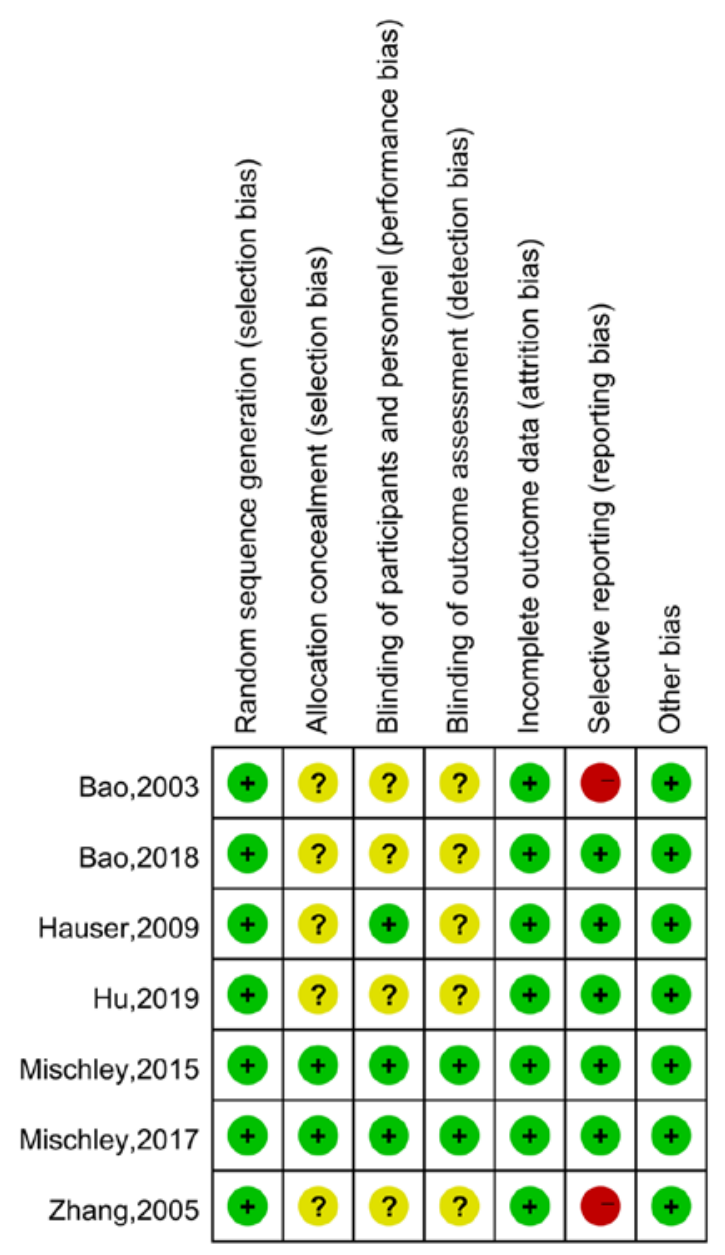

Figure 2. Risk of bias assessment; authors' judgments regarding each risk of bias item for each included study (summary).

studies. There were seven studies with a low risk of other bias. In conclusion, the overall quality of the seven included studies was moderate.

\section{Pooled results}

UPDRS. There were five studies reporting data on UPDRS I, II and III $(10-12,14,16)$. Due to differences in data type (end value and end value minus baseline value), the SMD was applied to determine differences in the UPDRS I, II and III scores between the GSH and control groups. The heterogeneity test did not reveal any differences between studies reporting data regarding the UPDRS $\mathrm{I}\left(\chi^{2}=3.51, \mathrm{I}^{2}=0 \%, \mathrm{P}=0.74\right)$; thus, the fixed-effects model was used (Fig. 4A). In addition, heterogeneity between the studies that reported data on UPDRS II was low $\left(\chi^{2}=7.51, \mathrm{I}^{2}=20 \%, \mathrm{P}=0.28\right)$ and thus, the fixed-effects model was applied once again (Fig. 4B). However, the heterogeneity test indicated moderate differences between studies reporting data on UPDRS III $\left(\chi^{2}=14.95, \mathrm{I}^{2}=60 \%, \mathrm{P}=0.02\right)$, and therefore, the random-effects model was used (Fig. 4C). The pooled SMD was $-0.04(95 \% \mathrm{CI}=-0.25-0.16, \mathrm{P}=0.70)$ for UPDRS I, 0.03 (95\% CI=-0.17-0.24, P=0.77) for UPDRS II and -0.48 [95\% CI=-(0.88-0.08), $\mathrm{P}=0.02]$ for UPDRS III. These pooled results demonstrated that, compared with the control groups, GSH may slightly improve the motor scores of patients with PD.
GSH-Px. In total, four studies (14-17) presented GSH-Px data for the GSH and control groups. The SMD was used to estimate differences in GSH-Px between the two groups. The results of the pooled SMD are presented in Fig. 5. There was significant heterogeneity among the studies $\left(\chi^{2}=72.36\right.$, $\left.\mathrm{I}^{2}=96 \%, \mathrm{P}<0.00001\right)$ and thus, the random-effects model was used. The pooled SMD was 1.88 (95\% $\mathrm{CI}=0.52-3.24$, $\mathrm{P}=0.007)$, indicating that compared with the control groups, serum GSH-Px levels were significantly higher in the GSH groups.

AEs. There were three studies reporting on the incidence of gastrointestinal reactions $(10,12,16)$; two papers containing data on dizziness or headache $(10,16)$; two articles reporting on involuntary movement $(10,16)$; two papers on labored breathing $(10,12)$; two articles presenting strep throatassociated data $(10,12)$; and two studies reported on insomnia $(10,16)$ in the GSH and control groups (Table II). The heterogeneity test revealed no differences between the studies (separately, $\mathrm{I}^{2}=0,0,0,41,41$ and $0 \%$, respectively); thus, the fixed-effects model was applied. Separately, the pooled RRs were $0.78(95 \% \mathrm{CI}=0.28-2.14, \mathrm{P}=0.62), 0.99$ (95\% CI=0.28-3.49, $\mathrm{P}=0.99), 0.33(95 \% \mathrm{CI}=0.44-2.99, \mathrm{P}=0.32)$, 1.59 (95\% CI=0.29-8.59, $\mathrm{P}=0.19), 1.59$ (95\% CI=0.29-8.59, $\mathrm{P}=0.59)$ and $1.64(95 \% \mathrm{CI}=0.23-11.74, \mathrm{P}=0.62)$. These pooled results of AEs demonstrate that the use of GSH appears to be safe.

Subgroup analysis: Influence of GSH dosage on UPDRS III. In total, two studies $(11,12)$ reported data of UPDRS III with the use of GSH $(300 \mathrm{mg} / \mathrm{d})$ for PD and two papers $(11,12)$ included data on the use of GSH $(600 \mathrm{mg} / \mathrm{d})$ (Fig. 6). The heterogeneity test indicated minimal differences between these studies (individually, $\chi^{2}=1.06, \mathrm{I}^{2}=6 \%, \mathrm{P}=0.30$; and $\chi^{2}=2.87, \mathrm{I}^{2}=30 \%$, $\mathrm{P}=0.24$, respectively), and therefore, the fixed-effects model was used. The pooled SMDs were -0.67 [95\% CI=-(1.30-0.04), $\mathrm{P}=0.04]$ and $-0.16(95 \% \mathrm{CI}=-0.41-0.09, \mathrm{P}=0.21)$, respectively, suggesting that the dose (300 vs. $600 \mathrm{mg}$ ) was an influencing factor for UPDRS III. Therefore, it was conservatively hypothesized that in patients with PD, a 300-mg dose of GSH may be more effective than a $600-\mathrm{mg} / \mathrm{d}$ dose.

\section{Discussion}

To the best of our knowledge, the present study was the first meta-analysis to evaluate the efficacy and safety of GSH for the treatment of PD. The study provided medical evidence-based support for the effectiveness and safety of GSH. The results of the meta-analysis were as follows: i) GSH does not have the potential to improve mentality, behavior, mood or the ability to perform daily activities, but has the ability to slightly improve motor function in patients with PD; ii) compared with the control groups, serum GSH-Px levels were significantly higher in the GSH groups, though there was notable heterogeneity between the studies ( $\mathrm{I}^{2}=96 \%$ ); iii) GSH appears to be safe and, compared with the control groups, does not increase the rate of AEs; and iv) the dose of GSH (300 vs. $600 \mathrm{mg} / \mathrm{d}$ ) may be one of the factors influencing motor function in patients with PD.

GSH (an antioxidant) is a tripeptide formed by the dehydration condensation of cysteine, glycine and glutamic 
Random sequence generation (selection bias)

Allocation concealment (selection bias)

Blinding of participants and personnel (performance bias)

Blinding of outcome assessment (detection bias)

Incomplete outcome data (attrition bias)

Selective reporting (reporting bias)

Other bias
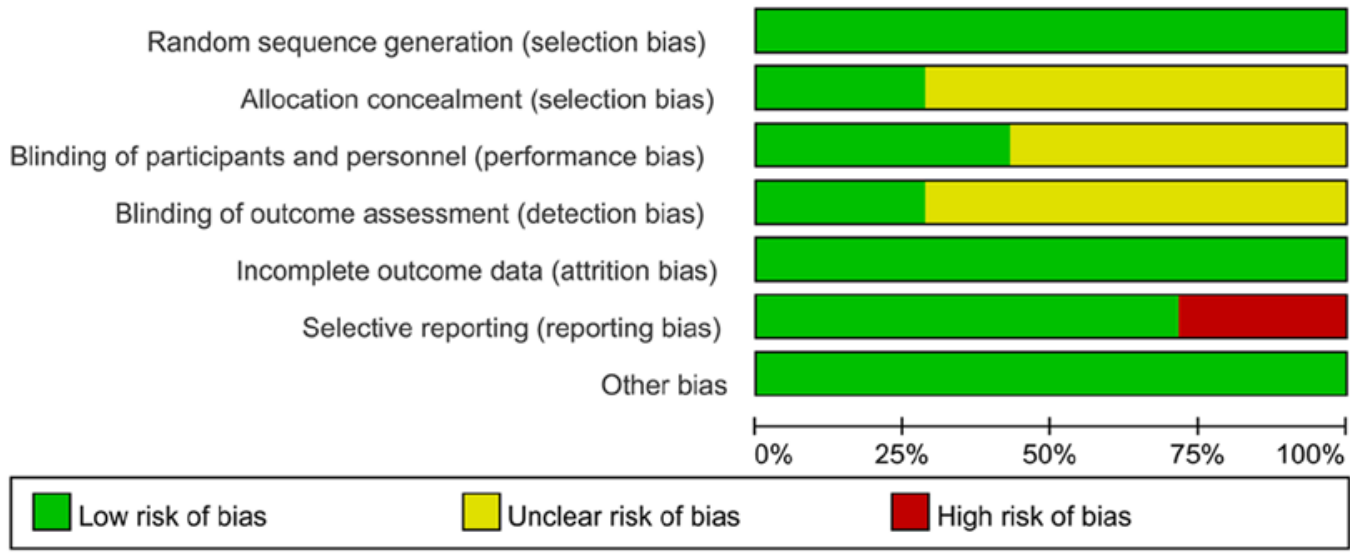

Figure 3. Risk of bias assessment, authors' judgments regarding each risk of bias item for each included study (graph).

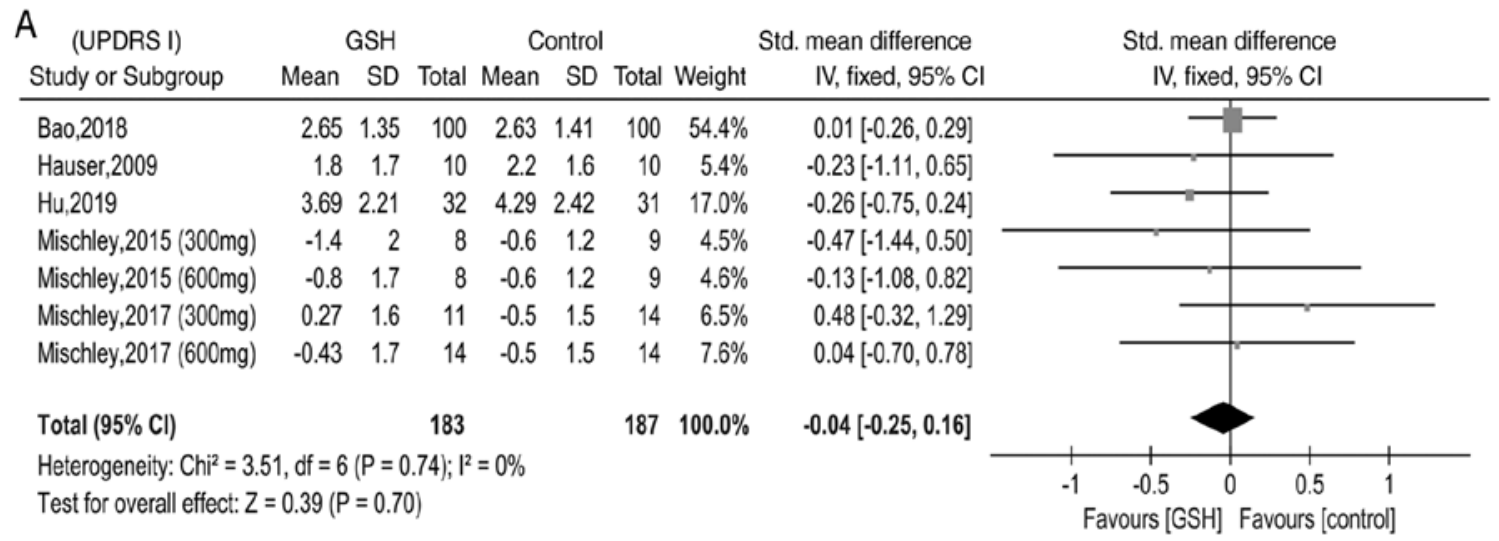

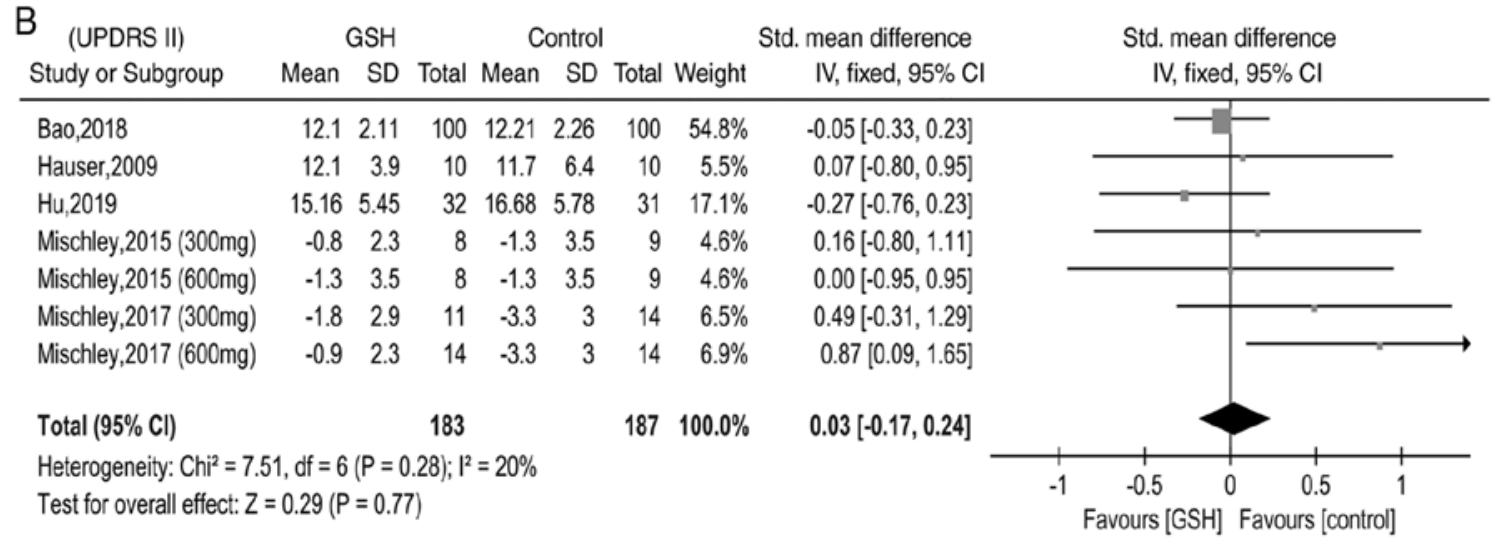

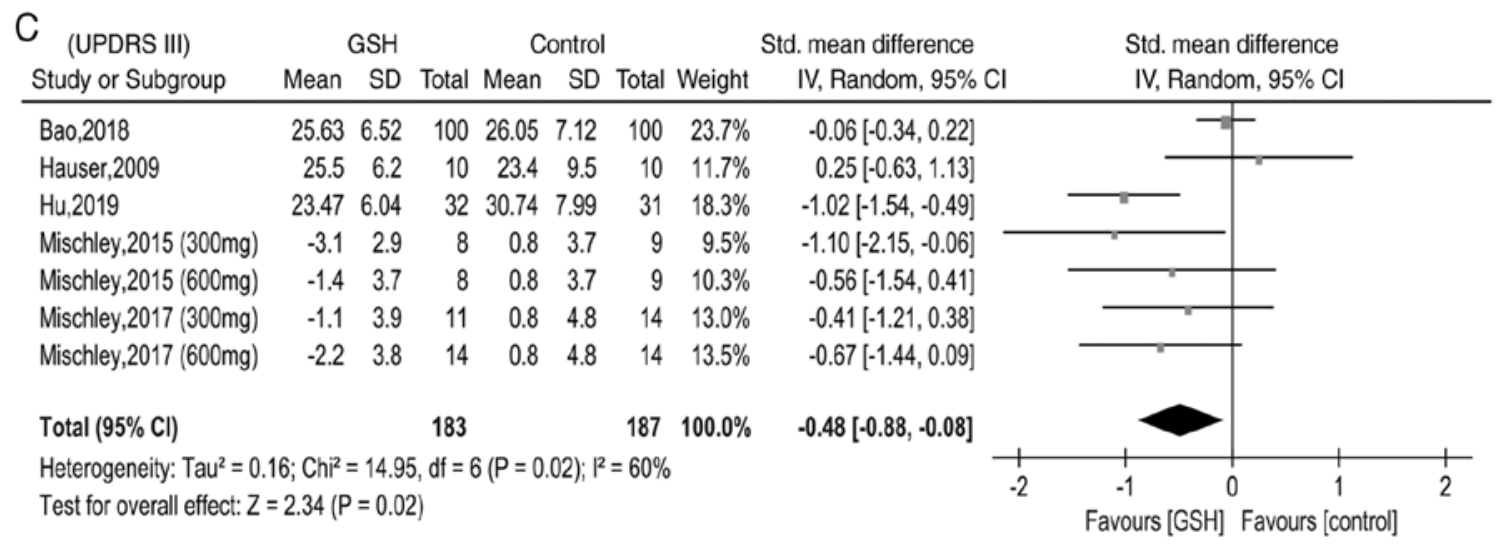

Figure 4. Meta-analysis of UPDRS between the GSH and control groups. (A) UPDRS I; (B) UPDRS II; (C) UPDRS III. UPDRS, Unified Parkinson's Disease Rating Scale; IV, inverse variance; df, degrees of freedom; SD, std. deviation; std., standard; GSH, glutathione. 
Table II. Meta-analysis of adverse effects compared between the GSH and control group.

Pooled results

\begin{tabular}{|c|c|c|c|c|c|c|}
\hline \multirow[b]{2}{*}{ Adverse effect } & \\
\hline & $\mathrm{RR}$ & $95 \% \mathrm{CI}$ & P-value & $\mathrm{I}^{2}(\%)$ & P-value & n-value \\
\hline Gastrointestinal reaction & 0.78 & {$[0.28,2.14]$} & 0.62 & 0 & 0.49 & 7 \\
\hline Dizziness or headache & 0.99 & {$[0.28,3.49]$} & 0.99 & 0 & 0.43 & 4 \\
\hline Involuntary movement & 0.33 & {$[0.44,2.99]$} & 0.32 & 0 & 0.99 & 4 \\
\hline Labored breathing & 1.59 & {$[0.29,8.59]$} & 0.19 & 41 & 0.19 & 5 \\
\hline Strep throat & 1.59 & {$[0.29,8.59]$} & 0.59 & 41 & 0.19 & 5 \\
\hline Insomnia & 1.64 & {$[0.23,11.74]$} & 0.62 & 0 & 0.59 & 4 \\
\hline
\end{tabular}

$\mathrm{N}$, number of study arms in the forest plot; RR, risk ratio.

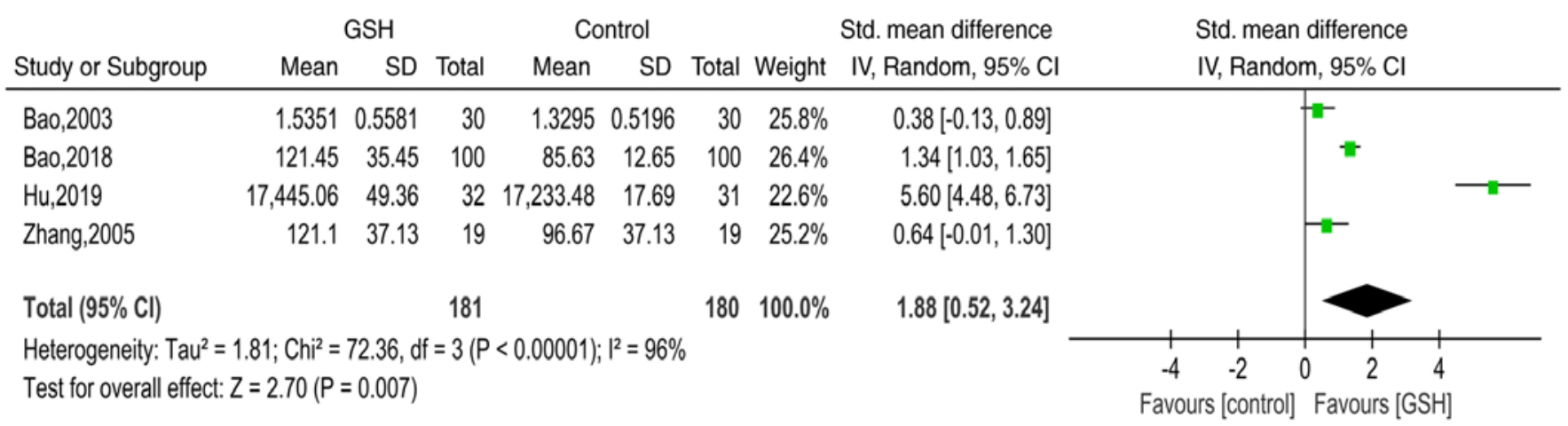

Figure 5. Meta-analysis of relevant studies assessing serum GSH-Px in Parkinson's disease. IV, inverse variance; df, degrees of freedom; SD, std. deviation; std., standard; GSH-Px, glutathione peroxidase.

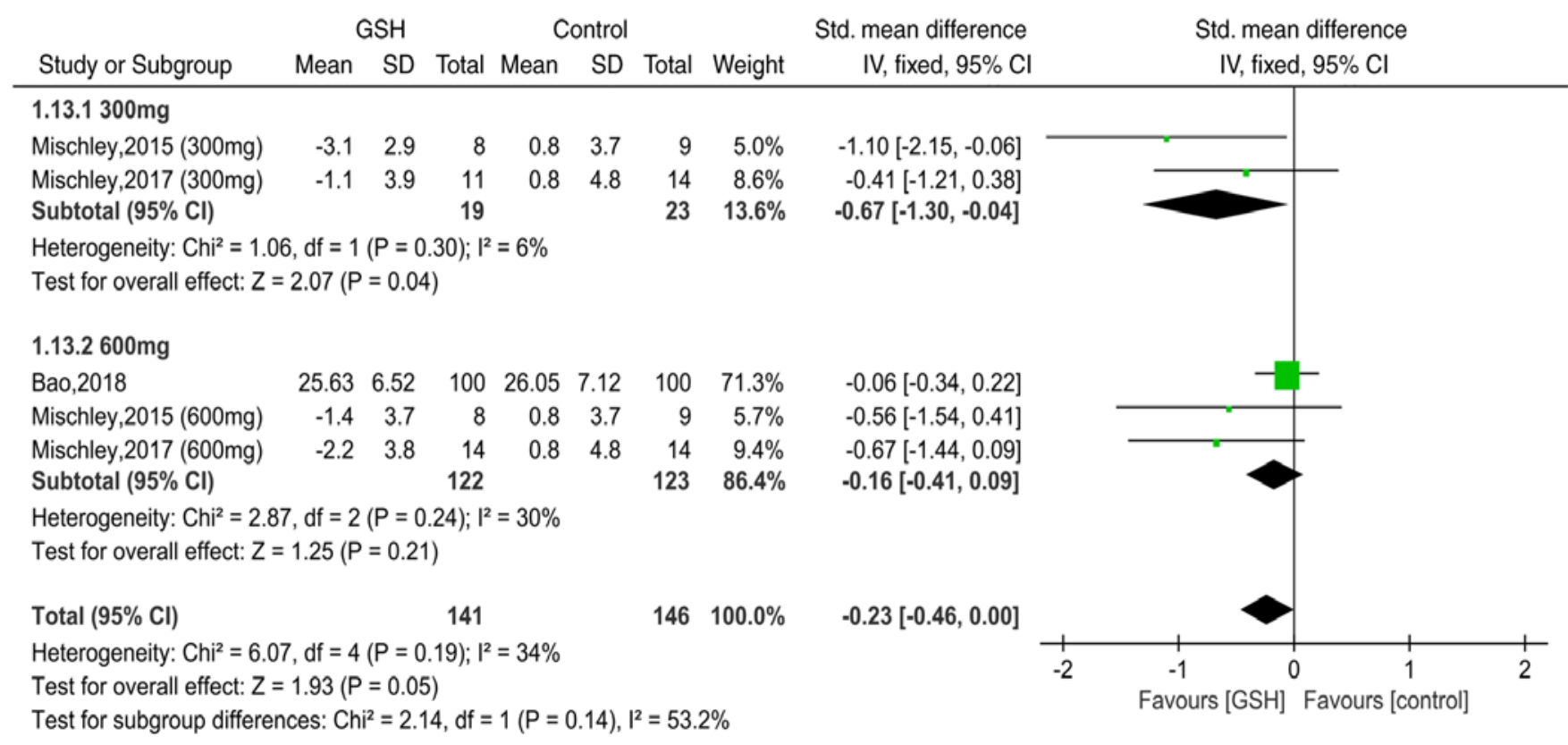

Figure 6. Subgroup analyses were performed in terms of the dose. IV, inverse variance; df, degrees of freedom; SD, std. deviation; std., standard; GSH, glutathione.

acid $(11,18)$. The tripeptide participates in redox reactions, which reduce damage to nerve cells caused by oxygen free radicals (11). Although most individuals synthesize sufficient
GSH to maintain a redox balance, this is not the case in patients with PD or other neurodegenerative diseases, which has been demonstrated to be associated with GSH consumption (11). 
Several studies have indicated that significant GSH depletion (30-50\%) is associated with an increased proportion of oxidized GSH in post-mortem PD substantia nigra tissues (19-21). Furthermore, a clinical study by Mischley et al (22) demonstrated that the whole-blood GSH concentration is negatively correlated with the clinical severity of PD. Furthermore, in vitro experiments have suggested that increased depletion of GSH results in selective impairment of mitochondrial complex I activity (23). To a certain extent, GSH replacement may provide symptomatic benefits to patients with PD by preventing mitochondrial dysfunction and thus reducing the impairment of dopaminergic function (10). In light of this, a series of clinical studies have been performed. In RCTs by Hauser et al (10), 21 subjects were randomly assigned to the GSH $(n=11)$ and control $(n=10)$ groups, which demonstrated that GSH is safe for use in patients with PD. However, there is currently no evidence to suggest that GSH is able to effectively improve the symptoms of $\mathrm{PD}$, which may be the result of the study sample being too small (10). In addition, Mischley et al $(11,12)$ also performed RCT studies, though the sample sizes of these studies were also small. In the present study, pooling data from Chinese and English studies revealed that GSH may mildly improve motor function in patients with PD. The results of several animal and clinical trials support these findings (24-26). However, although the present study provides preliminary medical evidence-based data on clinical studies, the effectiveness and safety of GSH supplementation requires further clarification.

Pooling the results of previous studies suggested that GSH-Px is positively associated with GSH levels. GSH non-enzymatically reacts with toxic free radicals and also acts as an electron donor in the reduction of peroxides catalyzed by GSH-Px (27). The resultant oxidized GSH is then being processed by GSH reductase and thus, GSH is recycled (27). GSH-Px has a major role in the recycling of GSH, which is supported by the fact that GSH-Px-knockout mice challenged with toxins (such as 1-methyl-4-phenyl-1,2,3,6tetrahydropyridine) exhibited greater dopamine depletion compared with age-matched control mice (28). Although the underlying mechanisms remain elusive, elevated GSH-Px concentrations appeared to be beneficial in alleviating the AEs of PD treatment.

In addition, several studies $(10,12,16)$ have reported data surrounding gastrointestinal reactions, dizziness or headache, involuntary movement, labored breathing, strep throat and/or insomnia. The pooled results of these studies revealed that the therapeutic dose of GSH is safe. Further patient studies also indicated that when GSH was repeatedly administered at doses of up to $5 \mathrm{~g}$ per day, both orally or intravenously, no toxicity was observed $(29,30)$.

In the present study, a subgroup analysis was used to identify the source of heterogeneity surrounding UPDRS III and to perform in-depth data mining. Subgroup analysis suggested that $300 \mathrm{mg} /$ day GSH was more effective than $600 \mathrm{mg} / \mathrm{d}$. However, it is worth noting that $300 \mathrm{mg} /$ day can not yet be confirmed as the optimal dose, because it is not known whether there are other possible optimal doses (the present study only compared the difference between 600 and $300 \mathrm{mg} /$ day). In addition, due to just a few studies with a relatively low population size reporting these data, particularly for $300 \mathrm{mg} / \mathrm{day}$, only two studies published by the same group with only 19 patients treated with GSH in total were included. Therefore, it was conservatively hypothesized that the administration of $300 \mathrm{mg} / \mathrm{d}$ or other doses of GSH warrants further investigation in future studies. Such research should be actively pursued in the future; animal experiments provide good evidence that GSH is not only safe but also potentially effective, though findings in humans require further clarification. The dose differences may be a cause of heterogeneity among studies. Furthermore, other confounding factors may also be a source of heterogeneity (such as ethnicity, sex, age, conventional treatment protocol, route of administration, course of disease and degree of disease severity).

The present meta-analysis has several limitations: i) Only seven articles comprising 450 patients were included and the quality of these articles was variable; ii) only studies published in the English and Chinese languages were included, which may have resulted in potential language bias; iii) due to data limitations, subgroup analysis by ethnicity, sex, age, course of disease and disease severity ere not performed; and iv) the pooled results warrant further clarification.

In conclusion, despite the limitations of the present study (which may have influenced these results), it was concluded that GSH may slightly improve the motor scores of patients with PD, though not at the expense of increased AEs. Furthermore, the GSH dosage may influence the efficacy. However, these conclusions warrant further investigation in the future.

\section{Acknowledgements}

Not applicable.

\section{Funding}

The work was supported by grants from the Six Talents Summit Training in Jiangsu Province (grant no. wsw-246), the Provincial Discipline Leader Category B (grant no. YZ201418501), the Jiangsu Province '13th Five-Year Plan' Special Fund for Science, Education and Health (grant no. RCC201807), the Jiangsu Province Key Experiments of Basic and Clinical Translation of Non-coding RNA (grant no. 201902) and the Jiangsu Province Natural Science Foundation (grant no. BK20190241).

\section{Availability of data and materials}

All data generated or analyzed during this study are included in this published article.

\section{Authors' contributions}

HLW, JZ, YPL, LD and YZC contributed to the interpretation of the data and writing of the paper. All authors read and approved the final manuscript.

\section{Ethics approval and consent to participate}

Not applicable. 


\section{Patient consent for publication}

Not applicable.

\section{Competing interests}

The authors declare that they have no competing interests.

\section{References}

1. Panicker N, Kanthasamy A and Kanthasamy AG: Fyn amplifies NLRP3 inflammasome signaling in Parkinson's disease. Aging (Albany NY) 11: 5871-5873, 2019.

2. Grespi F and Melino G: P73 and age-related diseases: Is there any link with Parkinson disease? Aging (Albany NY) 4: 923-931, 2012.

3. Tamano H, Nishio R, Morioka $\mathrm{H}$ and Takeda A: Extracellular $\mathrm{Zn}^{2+}$ influx into nigral dopaminergic neurons plays a key role for pathogenesis of 6-hydroxydopamine-induced Parkinson's disease in rats. Mol Neurobiol 56: 435-443, 2019.

4. Dorsey ER, Constantinescu R, Thompson JP, Biglan KM, Holloway RG, Kieburtz K, Marshall FJ, Ravina BM, Schifitto G, Siderowf A and Tanner CM: Projected number of people with Parkinson disease in the most populous nations, 2005 through 2030. Neurology 68: 384-386, 2007.

5. Smeyne M and Smeyne RJ: Glutathione metabolism and Parkinson's disease. Free Radic Biol Med 62: 13-25, 2013.

6. Soukup SF, Vanhauwaert R and Verstreken P: Parkinson's disease: Convergence on synaptic homeostasis. EMBO J 37: e98960, 2018.

7. Gaki GS and Papavassiliou AG: Oxidative stress-induced signaling pathways implicated in the pathogenesis of Parkinson's disease. Neuromolecular Med 16: 217-230, 2014.

8. Bisaglia M, Soriano ME, Arduini I, Mammi S and Bubacco L: Molecular characterization of dopamine-derived quinones reactivity toward NADH and glutathione: Implications for mitochondrial dysfunction in Parkinson disease. Biochim Biophys Acta 1802: 699-706, 2010.

9. Díaz-Hung ML, Yglesias-Rivera A, Hernández-Zimbrón LF, Orozco-Suárez S, Ruiz-Fuentes JL, Díaz-García A León-Martínez R, Blanco-Lezcano L, Pavón-Fuentes $\mathrm{N}$ and Lorigados-Pedre L: Transient glutathione depletion in the substantia nigra compacta is associated with neuroinflammation in rats. Neuroscience 335: 207-220, 2016.

10. Hauser RA, Lyons KE, McClain T, Carter S and Perlmutter D: Randomized, double-blind, pilot evaluation of intravenous glutathione in Parkinson's disease. Mov Disord 24: 979-983, 2009.

11. Mischley LK, Lau RC, Shankland EG, Wilbur TK and Padowski JM: Phase IIb study of intranasal glutathione in Parkinson's disease. J Parkinsons Dis 7: 289-299, 2017.

12. Mischley LK, Leverenz JB, Lau RC, Polissar NL, Neradilek MB Samii A and Standish LJ: A randomized, double-blind phase I/IIa study of intranasal glutathione in Parkinson's disease. Mov Disord 30: 1696-1701, 2015.

13. Higgins JPT and Green S (eds): Cochrane Handbook for Systematic Reviews of Interventions, version 5.1.0 (updated March 2011). The Cochrane Collaboration, 2011. http://training. cochrane.org/handbook.
14. Bao H: Clinical effect of reduced glutathione on Parkinson's disease. Chin J Clin Ration Drug Use 11: 44-45, 2018 (In Chinese).

15. Bao Y, Wang H, Chen H, Zhang B, Wang X, Xu G, Tong J, Wang Y and Yang X: An observation of 30 cases of Parkinson's disease treated with glutathiono. Anhui Med Pharmaceutical J 7: 22-24, 2003 (In Chinese).

16. Hu Y and Yang W: Clinical study of reduced glutathione in the treatment of Parkinsons disease. Chin J Pract Nervous Dis 22: 720-724, 2019 (In Chinese).

17. Zhang Y, Cao X, Hu H and Sun S: Therapeutic effect of reduced glutathione for Parkinson disease. Chin J Rehabil 20: 29-30, 2005 (In Chinese).

18. Aquilano K, Baldelli S and Ciriolo MR: Glutathione: New roles inredox signaling for an old antioxidant. Front Pharmacol 5: 196, 2014.

19. Fitzmaurice PS, Ang L, Guttman M, Rajput AH, Furukawa Y and Kish SJ: Nigral glutathione deficiency is not specific for idiopathic Parkinson's disease. Mov Disord 18: 969-976, 2003.

20. Pearce RK, Owen A, Daniel S, Jenner P and Marsden CD: Alterations in the distribution of glutathione in the substantia nigra in Parkinson's disease. J Neural Transm (Vienna) 104: 661-677, 1997.

21. Liddell JR and White AR: Nexus between mitochondrial function, iron, copper and glutathione in Parkinson's disease. eurochem Int 117: 126-138, 2018.

22. Mischley LK, Standish LJ, Weiss NS, Padowski JM, Kavanagh TJ, White CC and Rosenfeld ME: Glutathione as a biomarker in Parkinson's disease: Associations with aging and disease severity. Oxid Med Cell Longev 2016: 9409363, 2016.

23. Chinta SJ and Andersen JK: Reversible inhibition of mitochondrial complex I activity following chronic dopaminergic glutathione depletion in vitro: Implications for Parkinson's disease. Free Radic Biol Med 41: 1442-1448, 2006.

24. Sechi G, Deeden MG, Bua G, Satta WM, Deiana GA, Pes GM and Rosati G: Reduced intraveous glutathione in the treatment of ealy Parkinson's disease. Prog Neuropsychopharmacol Biol Psychiatry 20: 1159-1170, 1996.

25. Mischley LK: Glutathione deficiency in Parkinson's disease: Intranasal administration as a method of augmentation. J Orthomol Med 26: 32-36, 2011.

26. Zhou GH, Pao YC and Lu JM: The effect of glutathione on oxidation stress of Parkinson's disease rat. Chin J Behav Med Sci 13: 267-268, 2004 (In Chinese).

27. Bharath S, Hsu M, Kaur D, Rajagopalan S and Andersen JK: Glutathione,iron and Parkinson's disease. Biochem Pharmacol 64: 1037-1048, 2002.

28. Klivenyi P, Andreassen OA, Ferrante RJ, Dedeoglu A, Mueller G, Lancelot E, Bogdanov M, Andersen JK, Jiang D and Beal MF: Mice deficient in cellular glutathione peroxidase show increased vulnerability to malonate, 3-nitropropionic acid, and 1-methyl-4-phenyl-1,2,5,6-tetrahydropyridine. J Neurosci 20: $1-7,2000$

29. Dalhoff K, Ranek L, Mantoni M and Poulsen HE: Glutathione treatment of hepatocellular carcinoma. Liver 12: 342-343, 1992.

30. Tedeschi M, De Cesare A, Oriana S, Perego P, Silva A, Venturino $\mathrm{P}$ and Zunino F: The role of glutathione in combination with cisplatin in the treatment of ovarian cancer. Cancer Treat Rev 18: 253-259, 1991.

This work is licensed under a Creative Commons Attribution-NonCommercial-NoDerivatives 4.0 International (CC BY-NC-ND 4.0) License. 